\title{
Fractionation of Styrene-Acrylonitrile Copolymer by Column Adsorption Chromatography
}

\author{
Shinya Teramachi \\ Department of Industrial Chemistry, Kogakuin University, \\ Nakano-cho 2665-1, Hachioji, Japan. \\ Hiromi ESAKI \\ Department of Applied Chemistry, Nagoya University, \\ Furo-cho, Chikusa-ku, Nagoya, Japan.
}

(Received December 23, 1974)

\begin{abstract}
Chemical composition distribution determinations of styreneacrylonitrile random copolymers by column adsorption chromatography and by thin layer chromatography were compared at equivalent experimental conditions, by fractionating a 50:50 mixture of two copolymer samples with different chemical compositions using mixtures of chloroform and methyl acetate. The chemical composition distribution curve calculated from the fractionation data by column adsorption chromatography was not separated into two parts corresponding to the original components. If a concentration gradient method was employed in column adsorption chromatography, the mixed sample could be separated into two parts having different compositions. However, the resolution was not enough and a remarkable reversal of chemical composition was observed. On the other hand, the copolymer samples were separated according to their AN contents by thin layer chromatography. A discussion on the low resolution of column adsorption chromatography in comparison with thin layer chromatography is given.

KEY WORDS Column Adsorption Chromatography / Compositional Fractionation / Styrene-Acrylonitrile Copolymer / Thin Layer Chromatography / Molecular Sieve /
\end{abstract}

Random copolymers, in general, have distributions of chemical composition as well as molecular weight. The compositional distribution is an important factor for physical properties of copolymer samples. Therefore, it is desirable to establish a rapid and automatic method for the determination of chemical composition distribution. Several methods, such as fractionation, turbidity measurement, thin layer chromatography (TLC), light scattering measurements in several solvents, density gradient sedimentation, etc. have been proposed. Among them, cross fractionation $^{1-6}$ can give quantitatively reliable results but may be too laborious for practical polymer characterization. The TLC method ${ }^{7}$ is much easier than cross fractionation and also gives quantitative results. However, even this method cannot be operated as automatically as gel permeation chromatography for molecular weight distribution determination.

For rapid, automatic, and reliable determination of chemical composition distributions, the high-performance chromatography based on the same principle of adsorption-desorption as TLC may be most promising. Preparative column adsorption chromatography was first applied to SB rubber by Tagata and Homma. ${ }^{8}$ However, the reversal of chemical composition was found at the end of fractionation and the sample was not recovered quantitatively. The purpose of this work is to study the mechanism of column adsorption chromatography (CAC) by comparing it with TLC at equivalent experimental conditions, in order to improve the efficiency of CAC. 


\section{EXPERIMENTAL}

\section{Materials}

Copolymer samples used in the present work were styrene-acrylonitrile random copolymers prepared by bulk polymerization with benzoyl peroxide as initiator at $60^{\circ} \mathrm{C}$ under nitrogen atmosphere. These samples must have sharp distributions of chemical composition because of their low conversions and because their composition is near the azeotropic point of this polymer. Some commercial samples were also used in addition to them. Their acrylonitrile (AN) contents, limiting viscosity numbers, and conversions are summarized in Table I. The AN contents were determined by the microKjeldahl method and the limiting viscosity numbers were measured using a Ubbelohde-type viscometer in methyl ethyl ketone (MEK) at $25 \pm 0.01^{\circ} \mathrm{C}$.

Purification of MEK was carried out by distillation of first-grade reagent with the addition of $\mathrm{KMnO}_{4}$ under nitrogen atmosphere after drying with $\mathrm{Na}_{2} \mathrm{SO}_{4}$ and $\mathrm{K}_{2} \mathrm{CO}_{3}$. Firstgrade chloroform was purified by distillation after drying with $\mathrm{CaCl}_{2}$. First-grade methyl acetate was purified by distillation after drying with $\mathrm{MgSO}_{4}$. Other solvents used in TLC experiments were also first-grade reagents but were not purified.

The silica gel used as adsorbent was Wakogel C-200 obtained from Wako Junyaku Kogyo Co., Osaka, Japan; it had an average size of 200 mesh. The pore size distribution of the gel determined with a mercury pressure porosimeter (Carlo Erba Co., Italy) was shown in Figure 1. The diameters of the pores are distributed in the range from 100 to several hundred $\AA$. (Since the lower limit of pore diameter measurable is about $100 \AA$ with the present

Table I. Copolymer samples

\begin{tabular}{crcc}
\hline & \multicolumn{3}{c}{ AN content, $[\eta]$ in MEK } \\
wt $\%$ & at $25^{\circ} \mathrm{C}$ & $\begin{array}{c}\text { Conversion, } \\
\text { wt } \%\end{array}$ \\
\hline SA-1 $^{\mathrm{a}}$ & About 15 & - & - \\
2 & 22.7 & $1.38_{2}$ & 19.0 \\
3 & 27.2 & $1.39_{3}$ & 22.2 \\
$4^{\mathrm{a}}$ & 31.2 & $0.78_{1}$ & -(High) \\
\hline
\end{tabular}

a Commercial products.

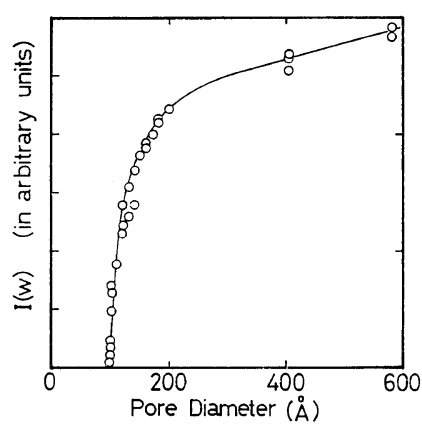

Figure 1. The integral distribution curve of diameter of silica gel used in column chromatography.

porosimeter, there may be pores having diameters smaller than this value.)

$T L C$

Developments of the copolymer samples having different compositions in TLC were carried out using several solvents and their mixtures. The TLC plate used was Replate-50 (Yamato Kagaku Kikai Co., Tokyo); its stationary phase layer was made by sintering a mixture of silica gel and glass powder on a glass plate. The thickness of the sintered layer was $0.2 \mathrm{~mm}$. Immediately before use, the layer was activated by heating the plate $110^{\circ} \mathrm{C}$ for $30 \mathrm{~min}$. Stock solutions for TLC tests were prepared by dissolving $50 \mathrm{mg}$ of each sample in $10 \mathrm{ml}$ of MEK. A spot of each solution, containing about $25 \mu \mathrm{g}$ of polymer sample, was formed at the point $2.0 \mathrm{~cm}$ from the end of the plate with a microsyringe. The plate was dried for several minutes and then the development was carried out at room temperature by the ordinary method. When the solvent front reached $10 \mathrm{~cm}$ from the starting point, the development was stopped by drying the plate in an oven at about $100^{\circ} \mathrm{C}$. The position of the polymer was visualized by spraying a methanol solution of iodine.

\section{Column Adsorption Chromatography}

In the experiments of CAC, the glass column used was maintained at $25^{\circ} \mathrm{C}$ by circulating constant temperature water through a glass jacket. The effective length and the inner diameter of the column were 80 and $4.2 \mathrm{~cm}$, respectively. The column was packed with 
silica gel activated by heating at $120-130^{\circ} \mathrm{C}$ for $2 \mathrm{hr}$ before use, using a slurry-packing method. That is, a slurry of silica gel and eluent, having suitable fluidity, was made in a beaker and poured into the column. The excess eluent on the top of the column was removed and an amount of eluent just enough to keep the top of the adsorbent wet was left. The height of the adsorbent in the column was about $40 \mathrm{~cm}$ in experiment $I$ and about $60 \mathrm{~cm}$ in the other experiments.

Twenty or thirty $\mathrm{m} l$ of the sample solution was poured into the column gently by using a pipet, that is, by keeping the tip of the pipet in touch with the inner wall of the column. Then the solution was allowed to flow until the upper level of the solution reached the top of the adsorbent. To elute out the copolymer, the eluent was stepwise added on the adsorbent in the same manner as the sample solution and flowed out from the bottom of the column. The rate of flow was adjusted by a stopcock at the bottom of the column. The fractions eluted from the column were collected in flasks.

Each fraction collected was concentrated by evapolating the solvents and then precipitated with methanol. The copolymer fractions thus obtained were washed with methanol and dried in vacuo at room temperature. Their weights, AN contents, and limiting viscosity numbers were measured in the same manner as those of the original samples.

\section{EXPERIMENTAL RESULTS}

\section{Results of TLC}

The $R_{f}$ values of three copolymer samples having different AN contents were determined by using eight solvents: MEK, tetrahydrofuran, chloroform, methyl acetate, ethyl acetate, $n$ propyl acetate, $n$-butyl acetate, and $n$-amyl acetate. The copolymers were all soluble in these solvents. The $R_{f}$ values determined are summarized in Table II. The table shows that the copolymer samples remain on the starting points or reach the solvent front without showing any intermediate $R_{f}$ values.

Mixtures of methyl acetate and chloroform with various compositions were selected for further experiments. The $R_{f}$ values obtained
Table II. Results of TLC tests

(Single solvent)

\begin{tabular}{llll}
\hline \multirow{2}{*}{ Solvent } & \multicolumn{3}{c}{$\begin{array}{c}R_{f} \text { Value } \\
\text { Copolymer sample }\end{array}$} \\
\cline { 2 - 4 } & \multicolumn{1}{c}{ SA-1 } & \multicolumn{1}{c}{ SA-2 } & SA-4 \\
\hline MEK & $1.0(0.68)$ & $1.0(0.76)$ & $1.0(0.76)$ \\
MeAc & $1.0(0.87)$ & $1.0(0.77)$ & $1.0(0.70)$ \\
EtAc & $1.0(0.82)$ & $1.0(0.85)$ & $1.0(0.81)$ \\
THF & $1.0(0.87)$ & $1.0(0.73)$ & $1.0(0.76)$ \\
$n$-PrpAc & $1.0(0.81)$ & $1.0(0.70)$ & 0 \\
$n$-BuAc & $1.0(0.82)$ & $1.0(0.74)$ & 0 \\
$n$-AmAc & $1.0(0.81)$ & $1.0(0.70)$ & 0 \\
Chloroform & 0 & 0 & 0 \\
\hline
\end{tabular}

( ), tail of spot.

Table III. Results of TLC tests

(Mixed solvent)

\begin{tabular}{|c|c|c|c|c|}
\hline \multirow[t]{2}{*}{$\mathrm{MeAc}: \mathrm{CH}_{3} \mathrm{Cl}$} & \multicolumn{4}{|c|}{$\begin{array}{c}R_{f} \text { Value } \\
\text { Copolymer sample }\end{array}$} \\
\hline & SA-1 & SA-2 & SA-3 & SA-4 \\
\hline $1: 20$ & 1.0 & 1.0 & 1.0 & 1.0 \\
\hline $1: 25$ & - & $1.0(0.85)$ & $0.90(0.55)$ & $0.80(0.40)$ \\
\hline $1: 30$ & - & $1.0(0.82)$ & $0.65(0.25)$ & $0.50(0.0)$ \\
\hline $1: 50$ & - & $1.0(0.79)$ & $0.52(0.32)$ & $0.50(0.0)$ \\
\hline $1: 100$ & - & $1.0(0.82)$ & $0.33(0.0)$ & $0.27(0.0)$ \\
\hline $1: 150$ & $1.0(0.76)$ & $1.0(0.40)$ & $0.16(0.0)$ & - \\
\hline
\end{tabular}

( ), tail of spot.

are summarized in Table III. It may be concluded that the present copolymers can be successfully fractionated by chemical composition in TLC without using a concentration gradient method, if these mixed solvents with intermediate compositions are used.

Results of $C A C$

The mixed solvents used in TLC were also examined for CAC of the same copolymers.

Three different series of experiments were carried out. In experiment I, elution was carried out with a mixed solvent having a fixed composition, whereas in experiment II elution was carried out by a concentration gradient method. In experiment III, the limiting viscosity number of each fraction, together with its chemical composition, was determined in order to examine the effect of molecular weight on CAC. 


\section{S. Teramachi and H. Esaki}

Table IV. Results of experiment I

\begin{tabular}{rrrc}
\hline $\begin{array}{c}\text { Fraction } \\
\text { no. }\end{array}$ & $w_{i}, \mathrm{mg}$ & $\begin{array}{c}\text { Weight } \\
\text { fract }\end{array}$ & $\begin{array}{c}\text { AN Content, } \\
\text { wt } \%\end{array}$ \\
\hline 1 & 9.2 & 0.121 & 17.8 \\
2 & 14.8 & & \\
3 & 1.8 & & \\
4 & 2.5 \\
5 & 15.8 & 0.101 & 23.9 \\
6 & 25.0 & 0.126 & 24.9 \\
7 & 26.4 & 0.133 & 24.9 \\
8 & 31.5 & 0.159 & 25.4 \\
9 & 29.3 & 0.148 & 25.2 \\
10 & 25.3 & 0.127 & 25.4 \\
11 & 16.8 & 0.085 & 15.1 \\
\hline Average & & & 23.3 \\
\hline
\end{tabular}

Original : 24.9

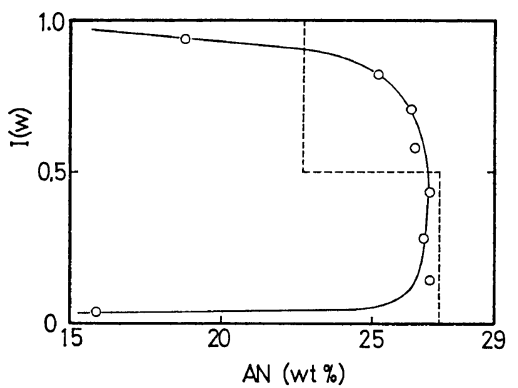

Figure 2. The integral chemical composition distribution curve calculated from the results in experiment I (Table IV).

In experiment $\mathrm{I}$, samples SA-2 of $0.1057 \mathrm{~g}$ and SA-3 of $0.1045 \mathrm{~g}$ were dissolved in $20 \mathrm{~m} l$ of a mixed solvent of methyl acetate and chloroform with volume ratio $1: 30$. The average AN content of the mixed copolymer sample was $24.9 \mathrm{wt} \%$. The fractionation was carried out by eluting $2,965 \mathrm{ml}$ of the mixed solvent. The volume of each fraction was $200 \mathrm{ml}$ except the last fraction, whose volume was $965 \mathrm{ml}$. The rate of flow of the eluent was $200 \mathrm{ml} / \mathrm{hr}$. The recovery of the sample was $94.8 \%$.

The results are summarized in Table IV and Figure 2. The difference between the average AN content of the original sample and that calculated from the data of fractions was somewhat larger than the experimental error of the nitrogen analysis, but the difference is not serious. Almost all fractions have an AN content value intermediate to those of the two original components. Moreover, the fractionation data in Figure 2 was not separated into two parts corresponding to two components. The remarkable reversal of AN content is observed in the last fraction. It may thus be concluded that this method, using a mixed solvent with a fixed composition, is not applicable to chemical-composition distribution determination of styrene-acrylonitrile copolymers by CAC.

In experiment II, the concentration gradient method was employed. Samples SA-2 of $0.2528 \mathrm{~g}$ and SA-3 of $0.2538 \mathrm{~g}$ were dissolved in $30 \mathrm{~m} l$ of chloroform. The elution was started from pure chloroform and the concentration of

Table V. Results of experiment II

\begin{tabular}{|c|c|c|c|}
\hline $\begin{array}{c}\text { Fraction } \\
\text { no. }\end{array}$ & $w_{i}, \mathrm{mg}$ & $\begin{array}{l}\text { Weight } \\
\text { fract }\end{array}$ & $\mathrm{AN}$, wt $\%$ \\
\hline 0 & 0 & - & \\
\hline 1 & 0 & - & \\
\hline 2 & 0 & - & \\
\hline 3 & 8.7 & 0.019 & 23.7 \\
\hline 4 & 70.9 & 0.152 & 24.7 \\
\hline 5 & 37.4 & 0.080 & 24.7 \\
\hline 6 & 12.9 & 0.028 & 24.5 \\
\hline 7 & 15.5 & 0.033 & 24.1 \\
\hline 8 & 17.9 & 0.038 & 24.0 \\
\hline 9 & 11.8 & 0.025 & 21.5 \\
\hline 10 & 9.8 & 0.021 & 18.7 \\
\hline 11 & & & \\
\hline $\left.\begin{array}{l}12 \\
13\end{array}\right\}$ & 21.1 & 0.045 & 21.7 \\
\hline 14 & 13.8 & 0.030 & 22.5 \\
\hline 15 & 23.6 & 0.051 & 25.5 \\
\hline 16 & 35.3 & 0.076 & 26.2 \\
\hline 17 & 64.4 & 0.138 & 26.5 \\
\hline 18 & 49.3 & 0.106 & 26.4 \\
\hline 19 & 26.3 & 0.056 & 26.5 \\
\hline 20 & 11.8 & 0.025 & 26.2 \\
\hline 21$)$ & \multirow{6}{*}{17.3} & \multirow{6}{*}{0.037} & \multirow{6}{*}{22.8} \\
\hline 22 & & & \\
\hline 23 & & & \\
\hline 24 & & & \\
\hline 25 & & & \\
\hline 26$)$ & & & \\
\hline 27 & 18.5 & 0.040 & 22.5 \\
\hline Average & & & 24.4 \\
\hline
\end{tabular}

Original : 24.9

Polymer J., Vol. 7, No. 6, 1975 


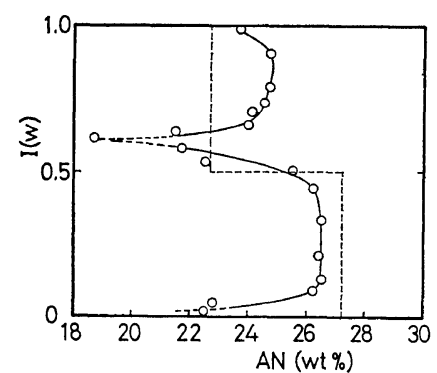

Figure 3. The integral chemical composition distribution curve calculated from the results in experiment II (Table V).

methyl acetate in the eluent was increased from 0 to 10 volume \% stepwise. The volume of each fraction was about $150 \mathrm{ml}$. The last (27th) fraction was obtained by using $1,000 \mathrm{ml}$ of solvent having 20 volume $\%$ of methyl acetate. The total volume of the eluent was $5,050 \mathrm{ml}$. The rate of flow was $270 \mathrm{ml} / \mathrm{hr}$. The recovery of the sample was $92.1 \%$.

The results are shown in Table $\mathrm{V}$ and Figure 3. The average AN content calculated from the fractionation data agrees with that of the original sample within experimental error. The chemical composition distribution curve shown in Figure 3 is composed of two distinct parts. However, the chemical compositions of the two parts are closer than those of the original two components though they are observed separately. Moreover the reversal of AN content appears both in the middle and the end fractions.

In experiment III, the sample solution was prepared by dissolving SA-2 of $0.5055 \mathrm{~g}$ in $30 \mathrm{ml}$ of a mixed solvent with volume ratio $1: 33$. The same mixed solvent of $5,500 \mathrm{~m} l$ was used as eluent. The volume of the last fraction was $1,000 \mathrm{ml}$ and the others were $300 \mathrm{ml}$. The rate of flow was $270 \mathrm{ml} / \mathrm{hr}$. The recovery of the sample was $94.3 \%$.

The results are summarized in Table VI and Figure 4. The limiting viscosity number of each fraction decreases in the order of fraction number. It may be concluded from this experimental result that there exists a molecular sieve effect in the CAC. This result is reasonable since the diameters of the micropores of the present silica gel shown in Figure 1 are comparable to the molecular dimensions of the
Table VI. Results of experiment III

\begin{tabular}{rrrrr}
\hline $\begin{array}{c}\text { Fraction } \\
\text { no. }\end{array}$ & $w_{i}, \mathrm{mg}$ & $\begin{array}{c}\text { Weight } \\
\text { fract }\end{array}$ & $\begin{array}{c}\text { AN } \\
\text { Content, } \\
\text { wt } \%\end{array}$ & $\begin{array}{c}{[\eta],} \\
100 \mathrm{~m} l / \mathrm{g}\end{array}$ \\
\hline 0 & 0 & - & - & - \\
1 & 29.6 & 0.062 & - & 1.486 \\
2 & 75.5 & 0.158 & 22.2 & 1.415 \\
3 & 12.1 & & & \\
4 & 1.9 & 0.058 & - & - \\
5 & 3.3 & & & \\
6 & 10.2 & & & 1.298 \\
7 & 43.2 & 0.091 & - & 1.110 \\
8 & 58.6 & 0.123 & 23.2 & 1.200 \\
9 & 77.2 & 0.162 & 23.7 & 1.145 \\
10 & 81.6 & 0.171 & 23.2 & 1.128 \\
11 & 50.7 & 0.106 & 23.0 & \\
12 & 15.1 & & & \\
13 & 0.4 & & & \\
14 & 4.4 & 0.069 & 22.4 & 0.770 \\
15 & 13.0 & & & \\
\hline
\end{tabular}

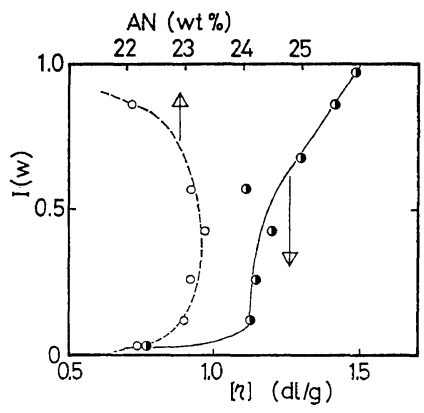

Figure 4. The integral distribution curves of chemical composition and limiting viscosity number calculated from the results in experiment III (Table VI):

$\bigcirc$, AN content; $\bigcirc$, limiting viscosity number.

present samples. That is, the fractionation of the copolymer is caused not only by chemical composition due to the adsorption-desorption mechanism but also by molecular weight due to the molecular sieve mechanism in CAC, whereas the effect of the molecular weight on TLC does not appear to be significant, in agreement with the results of Inagaki, et al. ${ }^{7}$ The reversal of AN content in the end fraction occurs in this experiment, too.

From the above experimental results, it is clear that the resolution power of compositional fractionation of CAC is lowere than that of 


\section{S. TeramaChI and H. Esaki}

TLC, not only when compared at equivalent experimental conditions, but also even when the concentration gradient method is employed in CAC.

\section{DISCUSSION}

The lower resolution power and the reversal of AN content are clearly due to the two effects, of adsorption-desorption and of molecular sieve, mentioned above. The present samples were prepared carefully by limiting the conversion within about $20 \%$ and the polymerization mixtures have compositions close to the azeotropic point. Nevertheless, a small deviation of chemical composition cannot be avoided. Moreover, the components having low molecular weight would have wider distributions of chemical composition than the components having high molecular weight. ${ }^{9}$ The components having higher molecular weights may not penetrate into the pores of the adsorbent, whereas the components having lower molecular weights may penetrate deeply into the pores. Moreover, the components of lower AN content in the pores may be eluted out from the pores more easily than the components of higher AN content due to the difference in their degrees of adsorption. In practice, it is observed in Figur 4 that the fraction showing the reversal of AN content has a lower molecular weight. This may also be a reason for the experimental result that the recoveries of the samples are not high and the average AN content calculated from the fractionation data of experiment $I$ is lower than that of the original sample.

A theory of the chromatographic separation of polymers under the double effects of molecular sieving and adsorbing was presented by White and Kingry. ${ }^{10}$ They showed by a model calculation that the reversal of molecular weight can occur, in a certain case, due to both effects. It may also be expected from their theory that the reversal of chemical composition may occur, in the chromatographic separation of copolymers, due to the same effects.

However, the present results do not necessarily mean that CAC always has a lower resolution power than TLC and that the molecular sieve effect cannot be avoided in CAC. There may be a possibility to improve CAC if we consider the reasons for the lower resolution power and the molecular sieve effect of CAC mentioned above. If adsorbents which have no pores comparable to the molecular dimensions of copolymer samples are used, the exact distributions of chemical composition might be obtained by CAC. It might also be possible to find solvent pairs which allow the separation by chemical composition without the influence of molecular weight in CAC.

The next question is why a molecular sieve effect is found in CAC, but not in TLC, when both experiments are carried out at equivalent experimental conditions. There are several studies concerning the molecular weight dependence of the $R_{f}$ value in TLC. Some author ${ }^{11-13}$ found a decrease of $R_{f}$ with molecular weight due to adsorption-desorption, but other authors ${ }^{13-16}$ found an increase of $R_{f}$ with molecular sieve mechanism and pointed out ${ }^{13,16}$ that both the adsorption-desorption and the molecular sieve mechanisms occur simultaneously. In the former group of experiments the adsorbents were kept dry before development, whereas in the latter the adsorbents were wet by pre-elution or by saturation of solvent vapor. Therefore our speculation is that there may exist both adsorption-desorption and molecular sieve mechanisms in TLC in general, but the molecular sieve mechanism may be more predominant if the activity of adsorbents is restrained by pre-elution and other processes. If the above speculation is true, it is understandable that no molecular sieve effect was found in TLC, but the effect was observed in column adsorption chromatography in the present work. This speculation is not in contradiction with the experimental results of Tagata and $\mathrm{Homma}^{8}$ who did not find the molecular sieve effect in CAC. In their case, the sample was deposited on the adsorbent by evaporating the solvent, so that the sample may have been adsorbed very strongly on the gel surface. However, too strong adsorption of polymers on the gel surface would result in unsatisfactory fractionation.

Acknowledgment. The authors wish to thank Professor Mitsuru Nagasawa of Nagoya University for his helpful discussions and 
Professor Michio Sekiya and Mr. Hiroshi Negishi of Kogakuin University for their help in the measurements of the porosity of the adsorbent.

\section{REFERENCES}

1. A. J. Rosenthal and B. B. White, Ind. Eng. Chem., 44, 2693 (1952).

2. A. D. Litmanovich and V. Ya. Shtern, J. Polym. Sci., Part C, 16, 1375 (1967).

3. V. A. Agasandyan, L. G. Kudryavtseva, A. D. Litmanovich, and V. Ya. Shtern, Vysokomol. Soedin., Ser. A, 9, 2634 (1967).

4. S. Teramachi and Y. Kato, J. Macromol. Sci.Chem., A4, 1785 (1970).

5. S. Teramachi and Y. Kato, Macromolecules, 4, 54, (1971).

6. S. Teramachi and T. Fukao, Polymer J., 6, 532 (1974).
7. H. Inagaki, H. Matsuda, and F. Kamiyama, Macromolecules, 1, 520 (1968).

8. N. Tagata and T. Homma, Nihon Kagaku Kaishi (J. Chem. Soc. Japan, Chem. Ind. Chem.), No. 7, 1330 (1972).

9. W. H. Stockmayer, J. Chem. Phys., 13, 199 (1945).

10. J. L. White and G. W. Kingry, J. Appl. Polym. Sci., 14, 2723 (1970).

11. E. P. Otocka and M. Y. Hellman, Macromolecules, 3, 362 (1970).

12. E. P. Otocka, ibid., 3, 691 (1970).

13. B. G. Belenkii and E. S. Gankina, $J$. Chromatog., 53, 3 (1970).

14. H. Halpaap and K. Klatyk, ibid., 33, 80 (1968).

15. E. P. Otocka, M. Y. Hellman, and P. M. Muglia, Macromolecules, 5, 227 (1972).

16. N. Donkai and H. Inagaki, J. Chromatog., 71, 473 (1972). 